\title{
Pazopanib in Primary Cardiac Angiosarcoma of the Right Atrium: A Case Report
}

\author{
Sophie Schur Rainer Hamacher Thomas Brodowicz \\ Comprehensive Cancer Center - Musculo Skeletal Tumors, GIST, Bone Sarcoma \& Soft \\ Tissue Sarcoma Program, Clinical Division of Oncology, Department of Internal Medicine \\ I, Medical University of Vienna, General Hospital Vienna, and Sarcoma Platform Austria, \\ Vienna, Austria
}

\section{Keywords}

Cardiac sarcoma $\cdot$ Angiosarcoma $\cdot$ Soft tissue sarcoma $\cdot$ Right atrium $\cdot$ Pazopanib

\begin{abstract}
Primary heart tumors are an extremely rare oncological entity with primary cardiac sarcomas usually representing $20 \%$ of all primary cardiac tumorous lesions [Shanmugam: Eur J Cardiothorac Surg 2006;29:925-932; Orlandi et al.: J Thorac Oncol 2010;5:1483-1489]. Angiosarcoma is the most prevalent histology and despite a multidisciplinary approach tends to have a dismal prognosis [Shanmugam: Eur J Cardiothorac Surg 2006;29:925-932; Fury et al.: Cancer J 2005;11:241-247]. Based on the prevailing literature, we report a 48-year-old woman diagnosed with primary metastatic cardiac angiosarcoma who showed a severe hypersensitivity reaction to conventional chemotherapy with taxanes but an excellent response to treatment with the multitargeted receptor tyrosine kinase inhibitor pazopanib.

$$
\begin{aligned}
& \text { C } 2016 \text { The Author(s) } \\
& \text { Published by S. Karger AG, Basel }
\end{aligned}
$$
\end{abstract}

\section{Case Report}

In October 2014, a 48-year-old woman was referred to the Department of Oncology at the Medical University of Vienna. Because of acute dyspnea, vertigo and precollaptic condi- 


\section{Case Reports in Oncology}

tion, the patient had been admitted to a peripheral emergency department 2 weeks before. Since pulmonary embolism was suspected, an immediate CT scan was performed, which showed multiple pulmonary nodules and a circumferential pericardial effusion. Subsequent transthoracic echocardiography confirmed a pericardial effusion of $3 \mathrm{~cm}$ with an enddiastolic compression of the right atrium and ventricle besides normal performance and size of the left ventricle.

Because of rapidly worsening clinical condition due to tamponade physiology, an emergency pericardiocentesis was done but the patient developed hemodynamic instability. Since iatrogenic trauma of the right atrium was suspected, the patient was transferred to the surgery department, where an emergency sternotomy had to be performed. During surgery, a 6 $\times 3 \mathrm{~cm}$ big right atrial mass was detected and subsequently entirely resected. The defect of the right atrium was restored with a nonvascularized pericardial autograft.

The patient's postoperative course was without any complications and a control echocardiography showed minimal pericardial effusion, a normal left ventricular systolic function as well as normal ejection fraction. Since histology revealed cardiac angiosarcoma (tumor cells stained positive for CD34), the patient was transferred to our oncology department for further examination and treatment.

Besides progressive multiple pulmonary nodules, a postoperative CT scan of the chest and abdomen showed no further distant metastases and no evidence of recurrence of the angiosarcoma in the right atrium. The patient had no prior medical history nor concomitant medication and after careful consideration, the patient was included in a clinical trial in which the combination of paclitaxel $70 \mathrm{mg} / \mathrm{m}^{2}$, days 1,8 and 15 each 28 days, plus pazopanib $800 \mathrm{mg}$ /day was applied.

During the first administration of paclitaxel at the end of November 2014, the patient suffered from severe hypersensitivity anaphylactic reaction with dyspnea, hypotension and chest pain despite intensified pretreatment with cortisone, diphenhydramine hydrochloride and ranitidine. An electrocardiogram revealed no cardiac problem and after additional i.v. corticosteroids, ranitidine and i.v. hydration, the patient's condition improved rapidly. According to the protocol, paclitaxel treatment was stopped and the patient continued with pazopanib $800 \mathrm{mg} /$ day monotherapy. Pazopanib was tolerated well with the exception of newly diagnosed arterial hypertension grade I-II, which, however, was efficiently treatable with oral medication.

A CT (thorax/abdomen) in January 2015 showed stable pulmonary disease without recurrence of cardiac angiosarcoma and the patient continued with pazopanib $800 \mathrm{mg} / \mathrm{day}$. In March 2015, a CT (thorax/abdomen) revealed partial remission of pulmonary metastases (reduction of both number and size of pulmonary metastases) and a new liver metastasis. Therefore, the patient was discontinued from the clinical study (as progressive disease according to RESIST occurred). Pazopanib was maintained (800 mg/day) and docetaxel was intended to be added. Despite intensified pretreatment with corticosteroids and antihistamines, the patient again suffered from severe hypersensitivity reaction and docetaxel was stopped immediately during the first application. However, pazopanib monotherapy (800 $\mathrm{mg}$ /day) was kept.

A subsequent CT (thorax/abdomen) in June 2015 showed a complete remission of all pulmonary metastases and a partial remission in the liver, both of which were confirmed in a CT (thorax/abdomen) in November 2015. The last CT scan in March 2016 revealed complete remission in the lungs and liver. Thus, pazopanib was continued (800 mg/day) and the patient still is in an excellent condition (ECOG 0). 
Schur et al.: Pazopanib in Primary Cardiac Angiosarcoma of the Right Atrium: A Case Report

\section{Discussion}

Primary heart tumors are an extremely rare oncological entity, occurring at a frequency of $0.11-0.30 \%$ in surgical series [1]. Importantly, $75 \%$ of all primary cardiac tumors are benign and only $25 \%$ malignant with primary cardiac sarcomas (PCS) usually representing $20 \%$ of all primary cardiac tumorous lesions $[1,2]$.

Angiosarcoma is the most prevalent histology accounting for $37 \%$ of all cases and is predominantly found arising from the right atrium [1,3]. Generally, primary cardiac angiosarcomas often afflict younger patients without predisposing factors and occur with nonspecific symptoms such as dyspnea, chest pain, cough, syncope, arrhythmias, fatigue or even life-threatening intracardiac obstruction $[4,5]$.

Despite a multidisciplinary approach, the overall prognosis of PCS remains poor with a median survival ranging from 11 to 18 months and a 5-year survival of $20 \%$ [2, 4]. Due to the rarity of the disease, the level of evidence for the optimal therapy algorithm of PCS is low [4]. Furthermore multimodal management is often extrapolated from extracardiac soft tissue sarcomas (STS), which, however, is problematic since cardiac surgery and radiation of dose-limiting organs like the heart and lungs are predominant features of PCS [4].

In elective cardiac sarcoma therapy, complete surgical excision remains the therapeutic mainstay, followed by a radio- and/or chemotherapeutic regimen, the latter preferably containing anthracyclines, ifosfamide or taxanes $[2,4,5]$.

Importantly, complete surgical resection is hardly achievable in PCS due to tumor inaccessibility and the vital role of cardiac structures, often requiring emergency surgery as described in our patient [4]. However, in a current retrospective series of 124 PCS patients of the French Sarcoma Group by Isambert et al. [4], surgery is a major prognostic factor for survival, regardless of the quality of resection as well as metastatic status. This may indicate that even incomplete resection appears worthwhile for immediate vital reasons as well as tumor control. The primary aim of surgery in terms of survival in metastatic PCS, however, is probably a more functional short-term outcome rather than prolonged survival and should only be considered in oligometastatic patients [4].

Radiotherapy with or without sequential chemotherapy has been traditionally performed in PCS despite the risk of myocardial injury owing to the limited tolerance of the heart to ionizing radiation [4]. Isambert et al. [4] reported that radiotherapy with a median dose of 50 Gy was performed mostly for patients with unresectable disease or in case of incomplete resection and was associated with better overall survival on univariate analysis and better progression-free survival on multivariate analysis. Since most PCS patients die of locoregional tumor progression, the trend for better overall survival with radiation therapy might be achieved through the improvement of locoregional tumor control [4].

Anthracycline-based chemotherapy regimens are still the gold standard for advanced and metastatic PCS and in the French case series, anthracycline-based chemotherapy was significantly associated with better overall survival, in the overall population and in nonmetastatic patients [4]. However, recommendation of systematic adjuvant chemotherapy seems difficult and recent data suggest that taxanes are more beneficial than anthracyclineifosfamide regimens in the treatment of angiosarcomas [4].

Primary cardiac angiosarcoma compared to other STS subtypes particularly prone to metastatic spread and survival outcome is described as even less favorable with an estimated median survival of 14 months $[4,5]$. For the treatment of primary cardiac angiosarcoma, radio- and/or chemotherapy are usually reserved for inoperable or metastatic disease or given to patients as adjuvant treatment for those with residual tumor [6, 7]. A multimodality 


\section{Case Reports in Oncology}

approach including radiotherapy and chemotherapy may only be effective for a selected subgroup of patients and radiotherapy dosage is limited by the sensitivity of lung and heart to radiation injury $[7,8]$.

Importantly, the well-known cardiotoxicity of anthracyclines can limit their use, especially in patients with intracardiac disease. Taxanes, as single agent or in combination with gemcitabine, have shown high antitumor activity in angiosarcomas and some evidence on the efficacy of taxanes in primary cardiac angiosarcoma has recently been accumulated [5, 9-11]. Furthermore, taxanes can be used as radio-sensitizing agents decreasing the adverse effects of high-dose standard fractionated radiotherapy especially in patients in favor of radical surgery $[9,10]$. Despite these data, the underlying mechanism of action of taxanes remains unclear. In vitro data suggest that taxanes may act as antiangiogenic agents in cardiac angiosarcomas [12], especially when administered weekly.

As suggested by the clinical course of our patient, we hypothesize a sustained antiangiogenic effect of pazopanib, a multi-targeted tyrosine kinase inhibitor, with activity against VEGF 1, 2 and 3 and platelet-derived growth factors [13]. Despite temporary progression in the liver, pazopanib was maintained and has led to a confirmed complete remission of lung and liver metastases. Thus, a maintained VEGFR inhibition beyond progression might be of value in angiosarcomas.

The clinical efficacy of oral pazopanib in patients with metastatic STS was demonstrated in a randomized, double-blind, placebo-controlled, phase III trial. In the multicenter PALETTE trial, pazopanib $800 \mathrm{mg}$ once daily significantly prolonged median progression-free survival compared to placebo (4.6 vs. 1.6 months) in adults with progressive metastatic STS following standard chemotherapy [14]. At final analysis of the PALETTE trial, median overall survival was $\approx 2$ months longer with pazopanib than with placebo, although this betweengroup difference was not statistically significant. Oral pazopanib was generally well tolerated with adverse events generally being grade 1 or 2 in severity [14] and therefore represents a valuable therapeutic option in cardiac angiosarcomas.

In conclusion, PCS in general and cardiac angiosarcoma in particular are rare and aggressive and require major therapeutic challenges. The poor prognosis of PCS warrants an extensive multidisciplinary approach as well as cohort studies to (I) promote earlier diagnosis, (II) achieve complete resection upfront and (III) improve local and systemic treatments towards the use of more personalized targeted therapies in specific histological subtypes. In order to guide treatment decisions for this complex malignancy, therapy should be tailored to each case and only be performed in specialized high-volume centers as the subject of a multidisciplinary consultation.

\section{Statement of Ethics}

Informed consent was obtained from the patient for publication of this case report.

\section{Disclosure Statement}

The authors declare that they have no competing interests. 


\section{Case Reports in Oncology}

\begin{tabular}{l|l}
\hline Case Rep Oncol 2016;9:363-367 \\
\hline $10.1159 / 000447088$ & $\begin{array}{l}\text { C } 2016 \text { The Author(s). Published by S. Karger AG, Basel } \\
\text { www.karger.com/cro }\end{array}$ \\
\hline
\end{tabular}

Schur et al.: Pazopanib in Primary Cardiac Angiosarcoma of the Right Atrium: A Case Report

\section{References}

2

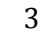

$>4$

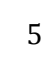

$-5$

$>6$

$>7$

$>8$

$>9$

10

11

$>12$

$-14$

Shanmugam G: Primary cardiac sarcoma. Eur J Cardiothorac Surg 2006;29:925-932.

Orlandi A, et al: Cardiac sarcomas: an update. J Thorac Oncol 2010;5:1483-1489.

Fury MG, et al: A 14-year retrospective review of angiosarcoma: clinical characteristics, prognostic factors, and treatment outcomes with surgery and chemotherapy. Cancer J 2005;11:241-247.

Isambert N, et al: Primary cardiac sarcomas: a retrospective study of the French Sarcoma Group. Eur J

Cancer 2014;50:128-136.

Minichillo S, et al: Efficacy of weekly docetaxel in locally advanced cardiac angiosarcoma. BMC Res

Notes 2015;8:325.

Penel N, et al: Phase II trial of weekly paclitaxel for unresectable angiosarcoma: the ANGIOTAX Study. J Clin Oncol 2008;26:5269-5274.

Elsayad K, et al: Primary cardiac angiosarcoma treated with positron emission tomography/magnetic resonance imaging-guided adaptive radiotherapy. Can J Cardiol 2016;32:829.e7-829.e10.

Potter R, et al: Primary angiosarcoma of the heart. Thorac Cardiovasc Surg 1989;37:374-378.

Nakamura-Horigome M, et al: Successful treatment of primary cardiac angiosarcoma with docetaxe and radiotherapy. Angiology 2008;59:368-371.

Suderman D, et al: Treatment of cardiac angiosarcoma with radiation and docetaxel: a case report with partial response and prolonged stable disease. J Thorac Oncol 2011;6:834-835.

Penel N, et al: Metastatic angiosarcomas: doxorubicin-based regimens, weekly paclitaxel and metastasectomy significantly improve the outcome. Ann Oncol 2012;23:517-523.

Pasquier E, et al: Antiangiogenic activity of paclitaxel is associated with its cytostatic effect, mediated by the initiation but not completion of a mitochondrial apoptotic signaling pathway. Mol Cancer Ther 2004;3:1301-1310.

Bramwell VH: Pazopanib and the treatment palette for soft-tissue sarcoma. Lancet 2012;379:18541856.

van der Graaf WT, et al: Pazopanib for metastatic soft-tissue sarcoma (PALETTE): a randomised, double-blind, placebo-controlled phase 3 trial. Lancet 2012;379:1879-1886. 